\title{
A wearable multi-site system for NMES-based hand function restoration
}

\author{
Andrea Crema, Nebojša Malešević, Ivan Furfaro, Flavio Raschellà, Alessandra Pedrocchi, and \\ Silvestro Micera IEEE Senior Member
}

\begin{abstract}
Reaching and grasping impairments significantly affect the quality of life for people who have experienced a stroke or spinal cord injury (SCI). The long-term well-being of patients varies greatly according to the restorable residual capabilities. Electrical stimulation could be a promising solution to restore motor functions in these conditions, but its use is not clinically widespread.

Here, we introduce the HandNMES, an electrode array (EA) for neuromuscular electrical stimulation (NMES) aimed at grasp training and assistance. The device was designed to deliver electrical stimulation to extrinsic and intrinsic hand muscles. Six independent EAs, positioned on the user forearm and hand, deliver NMES pulses originating from an external stimulator equipped with demultiplexers for interfacing with a large number of electrodes. The garment was designed to be adaptable to user needs and anthropometric characteristics; size, shape, and contact materials can be customized, and stimulation characteristics such as intensity of stimulation and virtual electrode location and size can be adjusted. We performed extensive tests with nine healthy subjects showing the efficacy of the HandNMES in terms of stimulation performance and personalization. Because encouraging results were achieved, in the coming months, the HandNMES device will be tested in pilot clinical trials.
\end{abstract}

Index Terms - NMES; hand; grasp; electrode array.

\section{INTRODUCTION}

$\mathrm{H}$ EALTHY subjects are capable of fine manipulation control with minimal apparent effort, even though grasping is a complex task that requires the coordinated action of several muscle groups. Natural human grasping is achieved by the synergistic activity of extrinsic, intrinsic, superficial, and deep muscles subjected to volitional control. Causes affecting grasp capabilities can vary, but as long as motor units are responsive to electrical stimulation, grasp assistance can be externally triggered. In particular, transcutaneous neuromuscular electrical stimulation (NMES) can be used to recruit different muscles in a coordinated way to restore grasping functions. However, NMES is not able to restore fine manipulation because it can selectively elicit only a limited and variable subset of muscles through the corresponding innervation points. First-generation NMES-based grasp assistive devices [1], [2] were able to achieve grasping by

A. Crema is with the Ecole Polytechnique Federale de Lausanne, Switzerland (e-mail: andrea.crema@epfl.ch).

N. Malešević is with Lund University, Sweden, and with the Faculty of Electrocal Engineering, Belgrade, Serbia. Ivan Furfaro and Flavio Raschella are recruiting mostly extrinsic flexors and extensors; in some cases, the stimulation of the thenar eminence was also used. These devices were characterized by very good usability, design simplicity, and mechanical robustness. Because of the use of large, non-selective electrodes and limited independent stimulation channels, these devices were able to induce coarse grasp patterns[3].

To increase the usability of this approach, stimulation has to be more selective and easier to personalize; therefore, the number of independent stimulation channels must increase. Such changes require a trade-off between effectiveness, technological complexity, and setup time. First-generation NMES systems used in parallel to achieve such functionality are prone to "spaghetti-cable" problems and are difficult to calibrate.

For these reasons, many groups have recently pursued new approaches (second-generation devices) to address the previously mentioned limiting factors, in particular, donning, tuning time, and limited functionality [4]-[8]. Lawrence and colleagues [9] developed an e-textile solution to improve wearability. Conductive threads, embroidered in the fabric, provide connectivity between skin electrodes and the stimulator. However, limitations apply to this design as conductive yarns are prone to wear and failure; redundant embroidery is needed to reduce the failure risk, and an isolating membrane coating is needed for electrical safety. To address this issue, Popović and colleagues [10] designed a generalpurpose thin electrode array (EA) using silver ink as a conductive medium. This technology grants better stability of the conductive lines while maintaining low linear impedance and thinner isolated lines, thus allowing for denser routing on flexible dielectric substrates.

Single channel NMES is known to be suboptimal and prone to fatigue [11] compared to physiological muscle recruitment. The use of EAs allows exploitation of spatial and temporal summation effects on the targeted anatomical structures. The studies of Nguyen and Malešević suggest the possibility of mitigating the exponential performance decay over time using distributed electrical stimulation sources[12]-[16].

In this paper, we present the HandNMES, a wearable device for NMES-based grasping restoration that is able to improve the performance of second-generation devices in terms of: (i)

with TNE Lab. EPFL. A. Pedrocchi is with Politecnico di Milano, Italy. S Micera is with Ecole Polytechnique Federale de Lausanne, Switzerland (e-mail: silvestro.micera@epfl.ch) and with Scuola Superiore Sant'Anna, Pisa, Italy. 


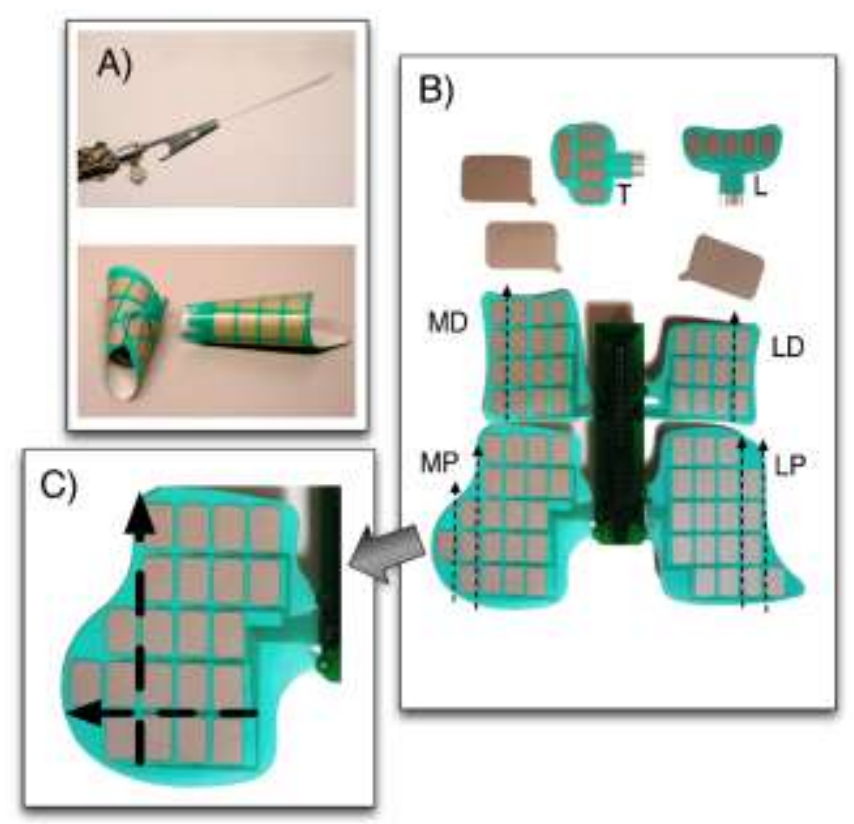

Fig. 1. A: Flexible electrode arrays have a paper-like touch and feel; the thickness, which does not exceed $150 \mu \mathrm{m}$, can be seen in the top image. In the same image block below are depicted two rolled electrode arrays fixed with a paperclip; the array on the right has a superimposed layer of gel (AG702, AmGel Technologies (®). The overall thickness of the matrices, gel included, is approximately $1 \mathrm{~mm}$. B: Of the six electrode arrays connected to the central $\mathrm{PCB}$, four of them constitute the butterfly-like body for extrinsic muscle stimulation. The electrode arrays are routed to allow trimming both in width and in length. This design allows reduction of the electrode arrays to fit subjects smaller than the maximum estimated size. C: Details of the routing can be seen in the bottom left callout box. All the arrays connected directly to the PCB are routed to allow external element trimming (top and lateral for top electrode arrays, bottom and lateral for bottom arrays). Hand electrode arrays, being mostly linear, do not require special routing to comply with functionality and adaptability.

wearability, by exploiting the advantages of the ink-based electrodes developed by Popović et al.; (ii) functionality, using EAs able to reduce fatigue and the time for selection of the stimulation parameters; and (iii) modularity, by allowing a personalized use of each EA.

To preliminarily verify the efficacy and usability of the HandNMES, the device was tested with healthy subjects in two experiments: open-loop by changing the stimulation parameters for each electrode to verify the flexibility of the system in terms of achievable joint movement and personalization; closed-loop to achieve grasping with desired force characteristics.

A further experiment was further conducted to show how stimulation selectivity plays a role in allowing different hand postures by differentiating grasps obtained single field stimulation versus sequenced multi-field stimulation.

\section{MATERIALS AND METHODS}

\section{A. Materials}

The EPFL local ethical committee approved the experiment. Each subject provided informed consent before proceeding with the tests. Nine healthy subjects ( 8 males, 1 female), aged between 23 years and 34 years, and homogeneous in forearm length, performed the tests. The garment was shortened to the chosen length by trimming the excessive electrodes. All the subjects used the same custom garment. To reduce the influence of gel degradation, new gel patches were used for every subject. Of the nine subjects, three were previously accustomed to NMES, and six subjects did not previously experience NMES and could thus be considered naive to such sensation.

\section{1) Garment for NMES}

In general, NMES responses exhibited significant intersubject and intra-subject variability from intrinsic notcontrollable parameters, such as different physiological conditions, electrode selectivity, skin stimulation filtering effects, tissue impedances and day-to-day variability, and different alignments between motor points and stimulation fields. In these experiments, we considered the size, shape, and position of the electrodes, as well as stimulation intensity and timing as design parameters that were tuned to optimize usability and the effectiveness of the system. To be able to target superficial muscles while maintaining comfort, we sized the active electrodes according to Kuhn and Lawrence [17]. Elongated electrodes are prone to forcing the injected charge distributions on the extremes of the main length axis; electrodes with sharp corners suffer the same problem and can produce a needle-like stimulation feeling. Round electrodes avoid this risk but when shaped in arrays do not efficiently cover the skin surface; they also pose the risk of not eliciting extremely selective and localized motor points (e.g., with extremely slender subjects). Larger electrodes mitigate the risk of high current densities and require a lower number of independent stimulation channels at the cost of reduced EA selectivity. In contrast, smaller electrodes increase the selectivity, the stimulator complexity, and the risk of higher current densities. To balance selectivity and electronic complexity, we chose to use two electrode sizes: $12 \mathrm{~mm}$ by $16 \mathrm{~mm}$ and $14 \mathrm{~mm}$ by 18 $\mathrm{mm}$. We arranged the electrodes in the array to fit subjects with large forearms and to be adaptable for fitting smaller subjects. The device was designed to fit a forearm length of up to 330 $\mathrm{mm}$ and a circumference in the proximal part of the forearm of up to $400 \mathrm{~mm}$. To customize the length and width of the device for various normotypes, the arrays were individually trimmed. The conductive paths routing from the connectors to the electrodes allowed the removal of unneeded electrodes without compromising the functionality of the internal electrodes.

As shown in Fig. 1, six EAs were included in the device: two were dedicated to activating intrinsic hand muscles (array L for lumbricals/palmar interossei and $\mathrm{T}$ for thenar eminence), two were for the extrinsic flexors (medial proximal MP and medial distal MD), and two were for the extrinsic extensors (lateral proximal LP and lateral distal LD). Three electrodes (PALS Oval $40 \mathrm{~mm}$ x $64 \mathrm{~mm}$, Axelgaard Manufacturing Co., LTD.) were used as anodes. The first was positioned on the anterior aspect of the wrist and coupled in combination with the MP, $\mathrm{MD}$ and $\mathrm{T}$ to elicit extrinsic grasp muscle responses and thenar muscle contractions. The second was located on the posterior aspect of the wrist and coupled with the LP and LD to elicit extrinsic muscles for finger openings. The third anode was positioned on the hand palm and coupled with the array L, positioned on the dorsal aspect of the hand, to elicit the contraction of intrinsic grasp muscles such as palmar interossei 


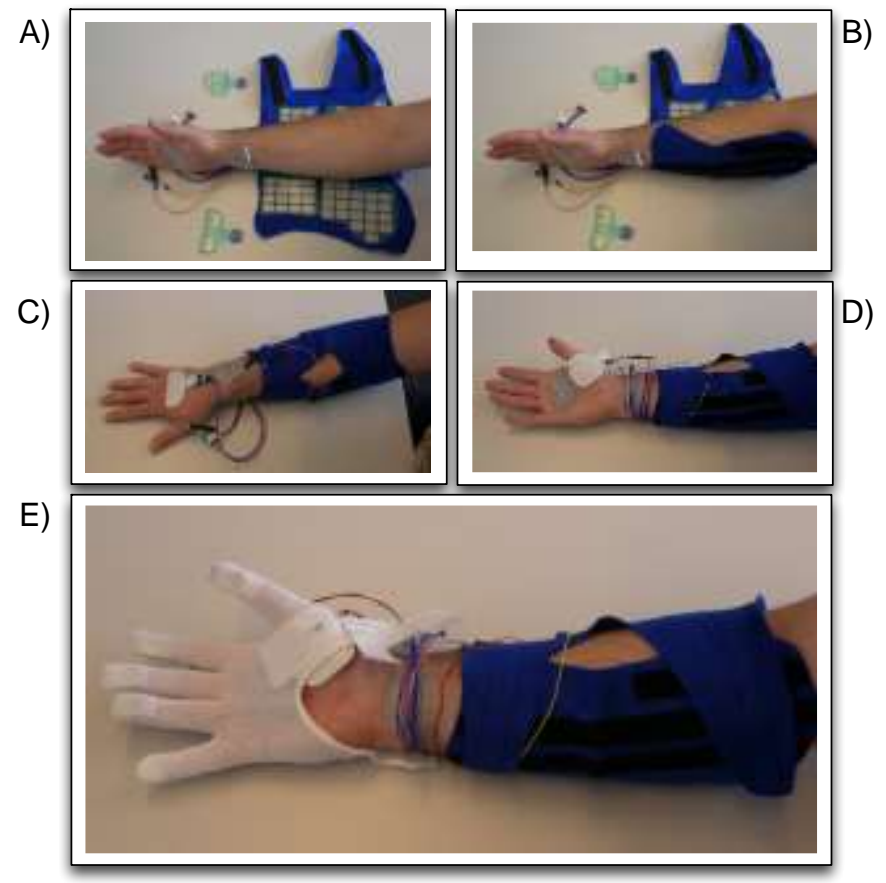

Fig. 2. A) Oval ground electrodes (Pals Clinical, Axelgaard Manufacturing Co., Ltd.) are positioned on the hand palm, on the anterior aspect of the wrist and on the posterior aspect of the wrist. B) The forearm, once aligned with the $\mathrm{PCB}$, has the most distal electrodes of the MD and LD arrays not closer than $2 \mathrm{~cm}$ from the corresponding counter electrodes. Each electrode array, which can be glued on the fabric to provide additional mechanical stability, is separately stuck on the subject's skin, and the garment is tightened with Velcro. C) and D) The small electrode arrays, stuck on the dorsal aspect of the hand and on the thenars, provide stimulation for intrinsic hand muscles. E) A cotton glove is used to protect the ground and hand electrodes during object manipulation. FSRs are hosted in sleeves to detect grasp and to provide grasp intensity information.

and lumbricals. A central rigid PCB, hosted under the subject's forearm, provided connectivity between the EAs and the electrical stimulator and limited the overall number of cables needed. The LD, LP, MP and MD arrays provided the butterflylike shape to the forearm garment. Plastic screws secured each array-PCB gate, providing mechanical stability against pulling and torsion. Polyester substrates with a thickness of $150 \mu \mathrm{m}$ isolated the conductive lines of the EAs; the substrate allowed the overall structure to be flexible (but not stretchable), conform to nearly flat or conical surfaces, and offer a paper-like touch and feel. The EAs contained a layer of glue to allow the fabric to adhere. The prototypes used either fake-leather fabric or felt fabric for hosting the PCB and the flexible EAs. Velcro hooks and straps tightened the device on the forearm. A removable gel patch placed on the EAs moderately increased the overall EA flexion stiffness. The gel patch contained AG702/735 gel (AmGel Technologies, Axelgaard Manufacturing) and was used to ensure good skin contact and for impedance matching. For simplified clinical usage and replacement of the disposable elements, gel patches were cut to match the shape of each EA. The gel patches provided higher adhesion on the EA side than on the skin side to allow complete and easy removal of the EAs from the skin. In previous approaches [5], [10], EAs were positioned on the skin on the approximate motor point position. Single patches were positioned using a garment, with anatomical landmarks used as absolute references. Fig. 2 shows the alignment process of the forearm and the garment. A cotton glove was used to secure electrode contact with the skin during object manipulation. Force-sensing resistors (FSRs, A201, A401, Tekscan Inc.) hosted in sleeves in the internal side of the glove detected grasp intensity information for the force feedback protocols.

\section{2) Stimulator}

When A customized version of the RehaStim stimulator with two DeltaStim demultiplexers (Hasomed GmbH, Magdeburg, Germany), developed by the manufacturer of the MUNDUS EU project [18], was connected to the HandNMES system. Stimulation was provided in time-frames of $50 \mathrm{~ms}$, each consisting of a volley of 10 independent pulses. Each pulse of the frame could be adapted in terms of intensity of stimulation, pulse width (PW), active electrode and counter electrode. The stimulator could communicate with a dedicated real-time Linux system with the Deltastim HART module [19].

\section{3) Motion capture}

Hand motion was recorded by an optoelectronic motion capture system (Vicon Bonita, Vicon Motion Systems Ltd. UK) with markers placed on the cotton glove. We created a hand model, derived from the Vicon standard model library, and used it to estimate the NMES induced motion. The model included wrist flexion-extension and ulnar-radial deviation. For the fingers, flexion of the metacarpal joint (MCP), the proximal interphalangeal joint (PIP), the distal interphalangeal joint (DIP), the thumb abduction, and the thumb opposition were recorded. Experiment protocol events provided by the graphical user interface (GUI) were synchronously recorded with the kinematic data as analog signals. Data were tracked and visually verified with Nexus software, low pass filtered and displayed with Matlab 8.3 (The MathWorks Inc., Natick MA). Data included in supplementary videos was recorded from Mokka [20].

\section{4) Instrumented glove for force detection}

A cotton glove (Safety s.p.a., Bovisio Masciago, Italy) was modified to host FSRs (Flexiforce A201-A401, Tekscan Inc., Boston, MA) in internal sleeves. A201 FSRs were placed on the fingertips, and one A 401 FSR was placed on the thenar eminence. An Arduino Mega 2560 (Arduino.cc) was used to sample and stream data via serial communication.

\section{5) GUI and sensory feedback}

To increase the usability of the device, the original communication protocol of the stimulator was ported to Labview (Labview 2013, National Instruments, Austin, Texas) 


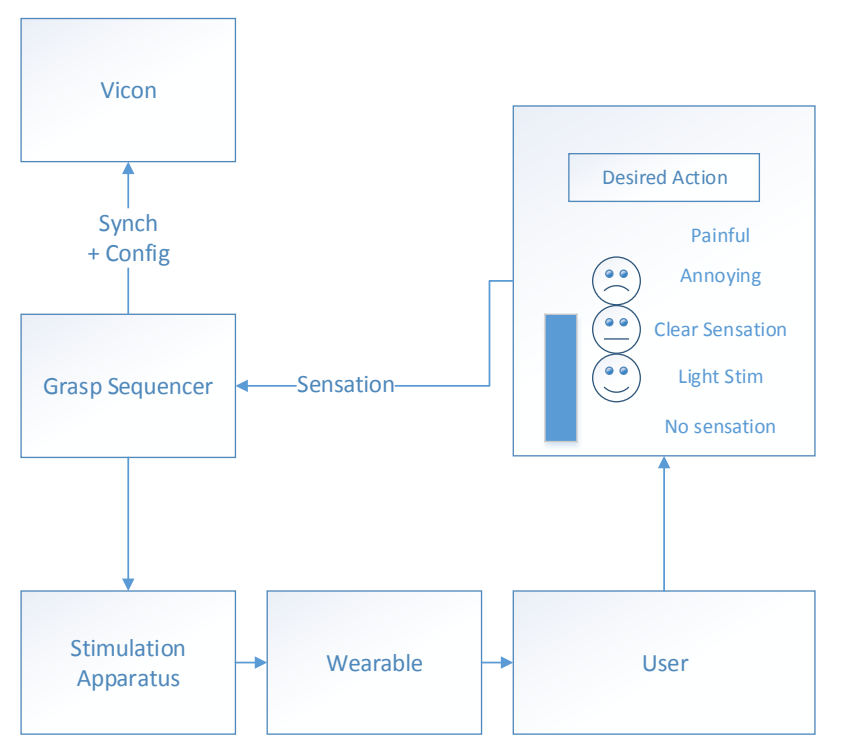

Fig. 3: Example of GUI for user feedback. The sensation is emoticon-coded both on the screen and on the keyboard. Visual and auditory messages on the expected next actions are used as reminders. The GUI allows specification of the targeted matrix and the boundary conditions for the stimulation scan procedure.

and custom GUIs were created. Experimental variables (array number, electrode number, phase of stimulation, current and PW intensity, and number of iterations) were sent to the analog input channel of the Vicon System with an analog-output board (NI9264, National Instruments, Austin, Texas), thus synchronizing the recording of kinematics and stimulation configurations. Multi-threading was used to handle two timed loops dedicated to control the stimulator and the analog output board, an event loop to detect the keyboard and GUI events, and one loop for handling experiment timing and phases (Fig. 3). An emoticon-coded keyboard was used to record the subject's sensory perception; buttons were conveniently mapped for left hand quick feedback. The subject selected "light sensation", "perceivable stimulation", "annoying feeling", and "painful sensation" to evaluate the received stimulation. The button encoding the "painful sensation" could also stop the stimulation current for the ongoing task. A minimalistic frame, always visible to the subject, ensured that the intended feedback was not mistyped.

\section{B. Methods}

Finding effective NMES parameters is primarily a tuning process. In this experiment, we considered three criteria to classify a stimulation configuration as valid: 1) induced finger movements have to be compatible with a chosen action or affordance without causing spasms in other districts, 2) wrist movements have to be compatible with a chosen action and should not deviate significantly from expected angles, and 3) stimulation should not cause adverse sensations. To be able to modulate grasp strength, we proceeded in two steps. First, the optimal parameters of stimulation in terms of location and current intensity were determined in the open loop. Second, we fixed the stimulation location and current intensity and closed the control loop by force feedback while using PW modulation to match the desired grasp intensity.

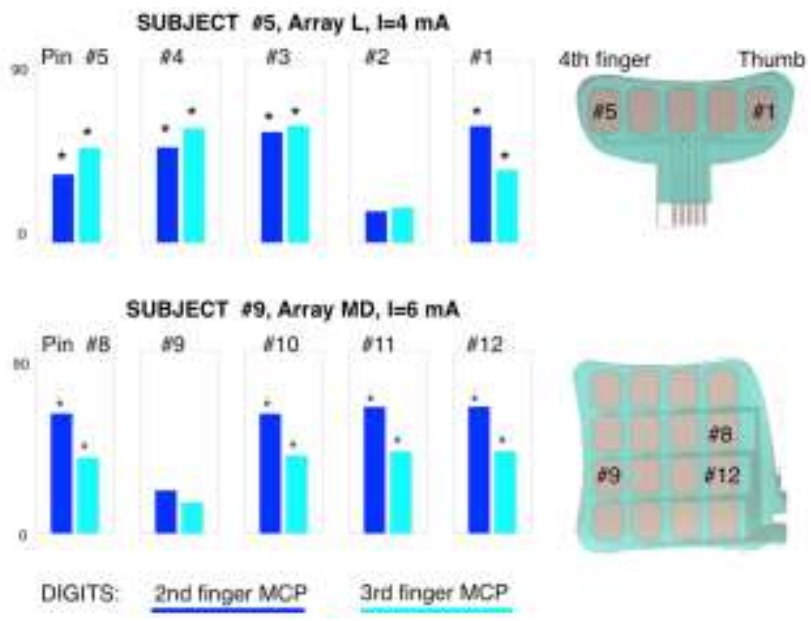

Fig. 4. Stimulation examples of two different arrays and detail of sequential scans on five pins. The metacarpal flexion of the middle finger (blue) and the ring finger (teal) are shown. During one pin stimulation cycle, variations of at least $10^{\circ}$ from the start angle to the end angle are used to discriminate induced motion from random passive motion; induced motion, active or passive, is marked with an asterisk. Top: the L array induces direct contraction of lumbrical and palmar interossei muscles. Stimulation on pin \#1 and \#2, close to the little finger side, mostly induces ring finger flexion, with the middle finger passively following, thus suggesting mostly ulnar nerve elicitation. As the stimulation location moves toward the center of the hand palm, on pin \#3, the middle and ring fingers are more elicited, and the flexion is higher and more balanced and suggests a purely median nerve stimulation. Pin \#4 stimulation does not induce motion, and the MCP joints stay in rest conditions. Pin \#5 causes a dominant flexion of the middle finger with passive flexion of the ring finger as a result of more medial branches of the median nerve. Bottom: the MD array induces contraction of the flexor digitorum superficialis of the flexor digitorum profundus. The sweep on the forearm produces a fast response to stimulation of the median nerve on pin 12 , which is able to effectively target the middle and ring fingers. The same selective motor response, but kinematically slower (not shown), is also clearly visible on pins 8,10 , and 11 where motor responses are suboptimal because of the distance

\section{1) Open loop characterization}

To characterize the selectivity that different electrodes can provide in terms of elicited kinematic responses, we stimulated one electrode at a time in each EA. In these tests, we targeted muscles with fine control and low innervation ratio. Stimulation frame rate was set at $20 \mathrm{~Hz}$, one pulse per frame enabled, and $\mathrm{PW}$ set to $250 \mu \mathrm{s}$ on extrinsic muscles. The PW was reduced to $150 \mu \mathrm{s}$ on hand intrinsic muscles because of better sensory tolerability of shorter pulses in these areas.

In these experiments, we achieved full sequential mapping by evaluating the local usefulness of stimulation. On each pin, we divided the task into three phases: A) stimulation that lasted 2 seconds; B) rest that lasted 2 seconds, during which the stimulation was off and the subject was asked to provide sensory feedback; and C) return that lasted 1 second, which was used to remind the subject to return to the initial task condition. When the task required stimulation of extensors, the initial condition was a relaxed closed hand. In contrast, when flexors were elicited, the hand had to be open in a relaxed state. The starting current intensity was set to $2 \mathrm{~mA}$, and the "stimulation", "rest" and "return" phases were iterated for all the pins in the chosen array. Once the sequential pin scan was completed, the current intensity was increased and the process was repeated. The current was increased in steps of $2 \mathrm{~mA}$ for matrices L, T, MP and MD and $4 \mathrm{~mA}$ for matrices LP and LD. The topological and intensity mapping was terminated when the stimulation was perceived by the subject as consistently annoying or painful, or when the task performance was expected not to improve 
because of an increase in the stimulation intensity. Fig. 4 shows a fraction of the sequential scan results for electrode arrays $\mathrm{L}$ and MD.

\section{2) Ranking methods}

Open loop identification data were ranked to find suitable stimulation locations and intensities to match the induced movements with a library of desired movements. Moreover, a set of stimulation parameters was considered valid if the stimulation did not cause discomfort. Ranking was calculated as the compound effect of the following criteria: limited wrist motion, effective grasping kinematics, and comfort of stimulation. It is possible to formalize this method as $J_{\text {global }}(i, k)=J_{w}(i, k) J_{f}(i, k) J_{s}(i, k)$ where $i$ represents the current intensity, $k$ represents the pin number of the tested electrode array, $J_{w}$ represents the wrist motion score, $J_{f}$ represents the finger joint movement score, $J_{s}$ is the sensory acceptability score, and $J_{\text {global }}$ is a measure of the performance of a stimulation pattern of the selected pin location and current intensity. All the parameters were normalized with " 1 " corresponding to an optimal response. When $\mathrm{J}_{\text {global }}$ exceeded an arbitrarily chosen threshold, we considered the stimulation pattern to be acceptable. As a fictitious example, let us assume a grasping stimulation with $\mathrm{J}_{\text {global }}=0.6, \mathrm{~J}_{\mathrm{s}}=1, \mathrm{~J}_{\mathrm{w}}=1$ and $\mathrm{J}_{\mathrm{f}}=0.6$. In this scenario, the stimulation was either not perceived or just above the perceptive threshold, the stimulation did not deviate the wrist from the neutral position, and at least one finger was flexing in a balanced way so that the MCP and the PIP joints were more than $50^{\circ}$ each. Score values of 0.6 were heuristically considered acceptable for arrays triggering extrinsic finger movements. Concerning $\mathrm{L}$ and $\mathrm{T}$ arrays, because of the higher hand sensitivity to stimulation and the fact that intrinsic hand muscles trigger mostly the MCP joints, a score of 0.3 was taken as a reasonable approximation. For each array, we considered the two best scores exceeding the threshold values to be the optimal parameter sets. Subcriteria for finger movements, wrist movements, and sensory acceptability are reported below. We assumed that the hand of the end-user was not constrained to make undesirable compensation schemes or mis-stimulation apparent. In such conditions, we wanted to avoid excessive wrist flexion-extension $\left(\vartheta_{F E}\right)$ and ulnar-radial $\left(\vartheta_{U R}\right)$ deviation. Therefore, we defined the maximum safety subspace as shown in Table 1. The wrist motion criterion was then defined as:

$$
\begin{aligned}
J(i, k)_{w}=I\left[\left(\vartheta_{F E}^{\min }\right.\right. & \left.\leq \vartheta_{F E}(i, k) \leq \vartheta_{F E}^{\max }\right)\left(\vartheta_{U R}^{\min } \leq \vartheta_{U R}(i, k)\right. \\
& \left.\left.\leq \vartheta_{U R}^{\max }\right)\right] *\left(1-\frac{1}{K_{\sigma 1}^{2}}\left(\vartheta_{F E}(i, k)-K_{\mu 1}\right)^{2}\right) \\
& *\left(1-\frac{1}{K_{\sigma 2}^{2}}\left(\vartheta_{U R}(i, k)-K_{\mu 2}\right)^{2}\right)
\end{aligned}
$$

where $K_{\mu 1}=\frac{\vartheta_{F E}^{\max }+\vartheta_{F E}^{\min }}{2}, K_{\mu 2}=\frac{\vartheta_{U R}^{\max }+\vartheta_{U R}^{\min }}{2}, K_{\sigma 1}=\frac{\vartheta_{F E}^{\max }-\vartheta_{F E}^{\min }}{2}$, $K_{\sigma 2}=\frac{\vartheta_{U L}^{\max }-\vartheta_{U L}^{\min }}{2}$, with I representing the indicator function. Angular deviations that exceeded the above described safety subspace were not allowed. The fingers criterion was defined as

$$
J_{f}(i, k)=1-\min _{i} \sum_{k} w\left(\vartheta_{i k}^{m}-\vartheta_{i k}^{d}\right)
$$

TABLE I

WRIST RANGE OF MOTION FOR

FLEXION-EXTENSION AND ULNAR-RADIAL DEVIATION

\begin{tabular}{ccccc}
\hline \hline Action & \multicolumn{2}{c}{ Ulnar-radial } & \multicolumn{2}{c}{ Flex-extension } \\
$\vartheta_{U R}$ & & \multicolumn{2}{c}{$\vartheta_{F E}$} \\
& & & & \\
\hline Open & $-30^{\circ}$ & $30^{\circ}$ & $0^{\circ}$ & $60^{\circ}$ \\
Close & $-30^{\circ}$ & $30^{\circ}$ & $-20^{\circ}$ & $40^{\circ}$ \\
\hline \hline
\end{tabular}

with $w$ representing a weight function for expected fingers movements, $\vartheta_{i k}^{m}$ the measured flexion-extension angle, and $\vartheta_{i k}^{d}$ the desired flexion-extension angle.

Finally, the sensory acceptability was determined as $J_{s}(i, k)=I(S(i, k) \leq 0.5)+(1-S(i, k)) I(S(i, k)>0.5)$.

The emoticon coded sensory feedback was assigned to the $S$ null value if no sensation was perceived, 0.25 if the subject perceived a light stimulation sensation, 0.5 if the sensation was clearly noticeable, 0.75 if the stimulation was annoying, and 1 when a painful sensation was elicited. The sensor acceptability criterion was designed to consider valid "light sensation" and "perceivable stimulation", to highly penalize "annoying feeling" reports, and to exclude any worse condition. The wrist motion was considered acceptable for opening and closing movements if the wrist motion was within a predefined flexextension range with no significant deviation on the ulnar or radial side. For hand opening, two possible conditions were considered valid: 1) fully extended digits from the index to little finger, or 2) the fully extended thumb. For hand closing, the possible conditions could be expressed as the opposite of the hand opening conditions. In such a way, because the motor point for thumb extension and the motor point for the extension of all the other digits were separated, superposition of stimulation effects could be taken as a simple approximation. For completeness, stimulation of the thumb extension could also trigger the index extension, which was remarkably less elicited by the activation of extrinsic extensors in general. The stimulation of extrinsic extensors, conversely, mostly affected motion of the ring, middle and little fingers. For hand closing movements obtained with the L, T, MP and MD arrays, a valid grasp was obtained if at least one finger was completely flexed on the MCP and PIP joints. The stimulation of intrinsic flexors could trigger complex behaviors of all the digits, but the effect consisted primarily of the simple flexion of the MCP joints close to the active pin, with no action of the corresponding PIP joints. Conversely, the stimulation of extrinsic flexors, depending on the depth of selective stimulation, could elicit either flexion or deviation of the wrist (superficial flexors); or induce the flexion of the PIP joints and assist flexion of the MCP joints and the wrist flexion (intermediate flexors); or induce flexion of the DIP joints while assisting PIP and MCP joint flexion. In all these cases, the predominant effect on the $\mathrm{PIP}$ and MCP was on the ring and middle fingers.

\section{3) Grasp sequencing synthesis}

As known, the human hand has more than 20 degrees of freedom. However, physiological and pathological constraints affect hand joints interdependence, and mechanical or controllability limitations reduce the overall degrees of freedom. As consequence the number of common hand postures is a limited subset [21]. Grasping real objects revolves around 

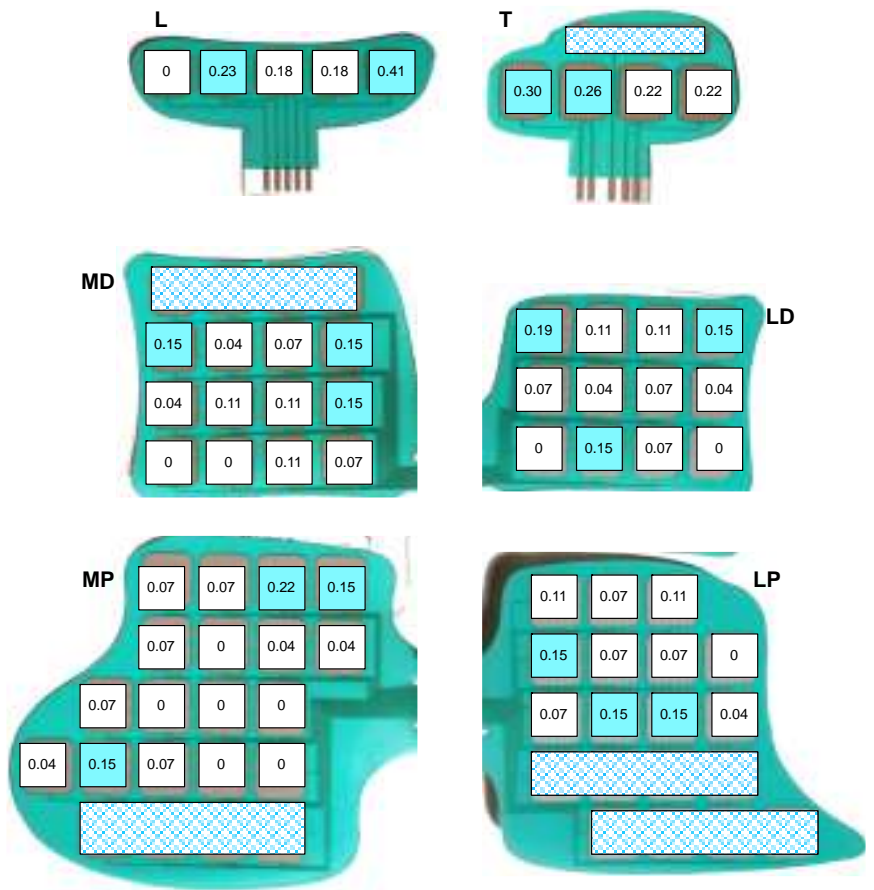

Fig. 5. Statistically probable location of useful motor points, normalized for each array. In each array, blue spots represent the ranked points that are optimal candidates for stimulation of the average subject. Such blue points are consequently the first points to be tested when calibrating the simulation for a new subject. The trimmed electrodes are represented as white rectangles with a teal grid.

the adaptation of the distal transverse, oblique and longitudinal arches to conform hand and fingers to the needed affordance [22]. Sollerman [23] and Light [24] reduced the overall grasps needed for activities of daily living to Power, Tip, Lateral, Spherical, Tripod, and Extension. NMES induced extrinsic flexors activation operates across the longitudinal arc, thus allowing to power grasp small cylindrical objects. Objects of larger diameter pose grasp consistency issues when only extrinsic flexors are used. Arnet et al. [25], [26] exemplify that, from a mechanical viewpoint, for functional grasping, the effect of extrinsic and intrinsic muscles activation need to be balanced to obtain functional kinematic trajectories. HandNMES can broaden the variety of hand postures by including selective sequential activation of intrinsic muscles. This allows to have selective single-finger or two-finger activation by using lumbrical muscles (matrix L) and thenar muscles (matrix T). To demonstrate such possibility, we first performed a sequential scan of the five L matrix active sites (test 1), one active site a time from index to ring, while keeping constant the stimulation intensity $(\mathrm{I}=12 \mathrm{~mA}, \mathrm{PW}=100 \mathrm{us})$. We repeated the same procedure with the matrix $\mathrm{T}$ (test 2 ) to identify the optimal active field for thumb opposition ( $\left.\mathrm{T}_{\mathrm{opp}}\right)$. We then modulated the sequenced activation of single active fields of the L matrix, and of $\mathrm{T}_{\mathrm{opp}}$ with a delayed onset of 4 seconds (test 3 ). In test four we modulated groups of active fields $\left(\mathrm{L}_{1+3}, \mathrm{~L}_{3+5}, \mathrm{~L}_{1+3+5}\right)$ and activated $\mathrm{T}_{\text {opp. }}$. Test five mimicked test four sequencing, but included a delayed activation of the extrinsic flexors and a synchronized deactivation of lumbricals.

\section{4) Closed loop control}

The RehaStim allowed current intensity increments of $2 \mathrm{~mA}$ from $0 \mathrm{~mA}$ to $110 \mathrm{~mA}$ and $\mathrm{PW}$ increments of $1 \mu \mathrm{s}$ from 50 $\mu \mathrm{s}$ to $500 \mu \mathrm{s}$. For comfort purposes stimulation was limited to up to $30 \mathrm{~mA}$, and pulsewidth from $50 \mu \mathrm{s}$ to $300 \mu \mathrm{s}$. The optimal current intensity and location of stimulation were taken from the ranking results of the open loop identification. The grasp force was controlled by PW modulation. The flat force sensor positioned in the thenar eminence of the glove (Fig. 2E) measured the grasp intensity with a $50 \mathrm{~ms}$ impulse response delay and long-term drift. The NMES muscle response finite delay was estimated to be approximately 200 ms. A proportional integral control with anti-wind-up was tuned for reducing the risk of spasm.

\section{RESULTS}

\section{A. Grasp eliciting and comfort}

Nine subjects that volunteered in this study found the stimulation acceptable during the preliminary stimulation phase and became accustomed to the stimulation sensation. Fig. 4 depicts the effects of selectivity from adaptable locations of stimulation and indicates that small location changes can provide current spreading effects, causing coarse stimulation. It is worth noting that finger movements were coupled, and consequently single finger movements could usually not be achieved with single motor point stimulation, whereas adjacent joints could passively follow the triggered finger movements.

Fig. 5 shows the global expectations for functional stimulation. For each array, we choose the best three stimulations on for each subject, described as stimulation's pin, depending on the value of $J_{\text {global }}$. The resulting matrix was normalized for all the subjects, providing a probability of useful motor point locations. It should be noted that intra-subject results can vary significantly, as shown in Fig. 6. Several causes in particular could account for this variability; e.g., variability among the subjects in terms of forearm circumference, different positions of the electrode array and physiological conditions during stimulation. As shown in Fig. 6, despite the homogeneity in terms of forearm length, subject \#4 on the left and subject \#6 on the right exhibited substantial differences in stimulation location, intensity of optimal currents, and acceptability of stimulation. With hand intrinsic muscles, current intensity effects were more noticeable, including lower relative current thresholds for subject \#4. This observation was generally consistent across the tests, with subject \#4 on average requiring lower stimulation than subject \#6 to obtain similar effects. The optimal stimulation locations produced detectable and partially overlapping patterns, as well as moderately different optimal locations, thus suggesting that a partial information transfer of stimulation maps from subject to subject; it is also possible (but not necessarily optimal) that such transfer occurs within the same subject but in different sessions. As an example, for array LP, subjects \#4 and \#6 exhibited similar J scores of 0.87 and 0.91, respectively, but the optimal locations of the two electrodes were axially shifted (by approximately $40 \mathrm{~mm}$ ).

As shown in Fig. 6, perceptive results of subjects \#2 and \#3 appear in generally darker shades than the ones observable with subjects \#4 and \#6. The $2 \mathrm{~mA}$ increase step of stimulation intensity in these subjects was relatively high for fine tuning of an acceptable motor response and for an acceptable global response. The optimal scores in this case also depended highly on the overall acceptability of the stimulation, which could 

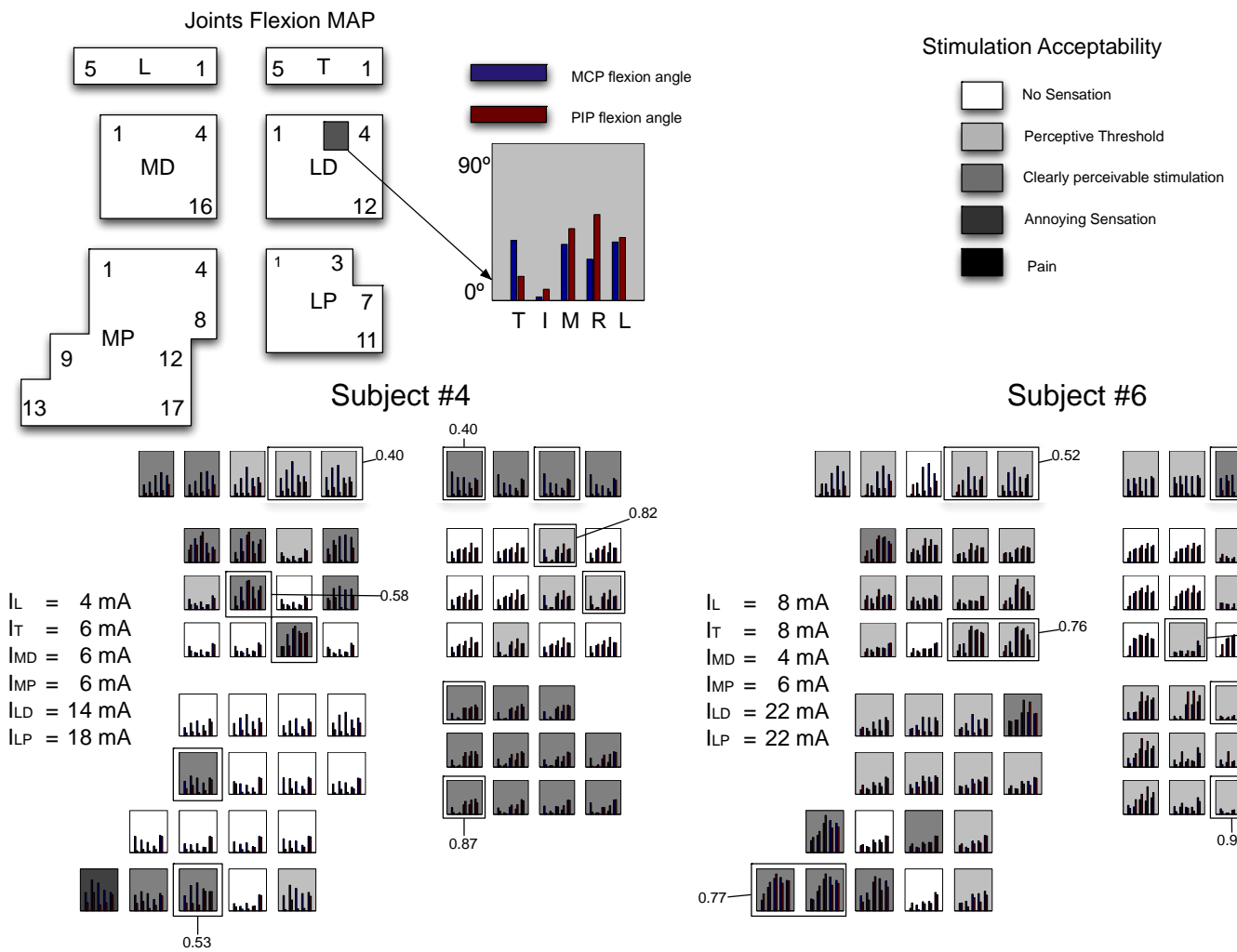

Subject \#2
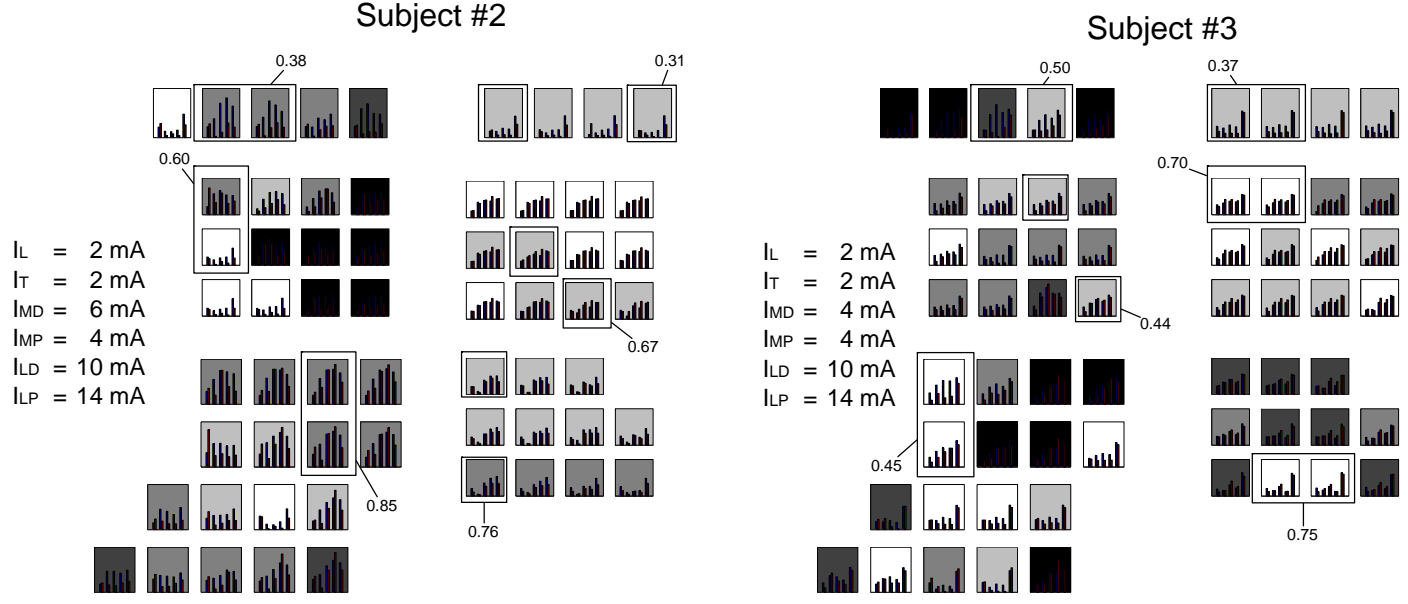

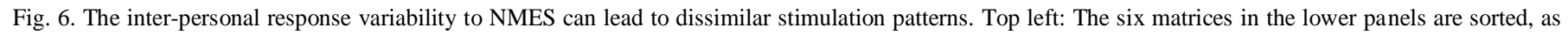

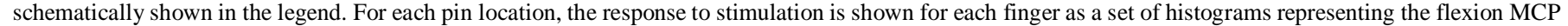

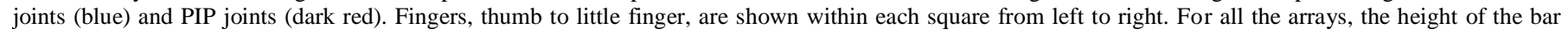

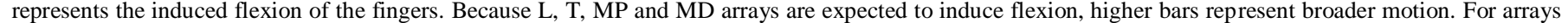

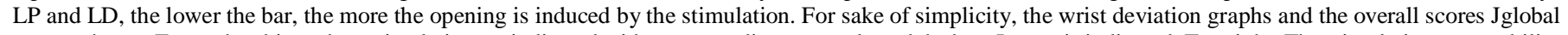

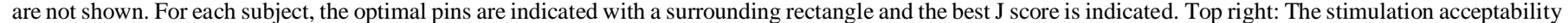

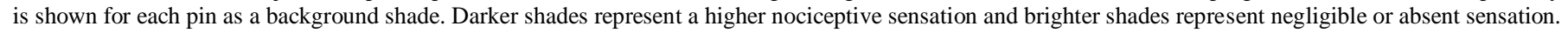

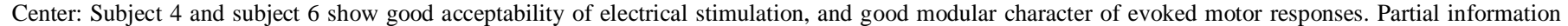

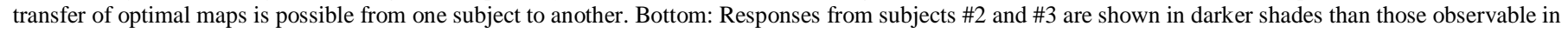
subjects \#4 and \#6, and proper tuning of a sensory acceptable motor response seems harder to achieve, with more sparse and globally lower optimal responses.

easily disqualify topologically close locations. As an example, subject \#3 obtained low optimal scores on array MP with an optimal $\mathrm{J}=0.45$.

\section{B. Grasp sequencing}

NMES elicited hand intrinsic muscles are not coupled with wrist motions and can provide higher fingers more selectivity than extrinsic muscles. Additionally, they are less subject to the relative motion of skin and underlying tissues that is observable e.g. during pronation-supination, which can affect selective stimulation of extrinsic flexors and extensors. The electrode arrays $\mathrm{L}$ and $\mathrm{T}$ and the corresponding counter electrodes are positioned as shown in Fig. 2C-D-E and in Fig. 7 to optimize the selectivity and stability of the contact. In this design, due to the stiffness of the material and to the continuous stress that 


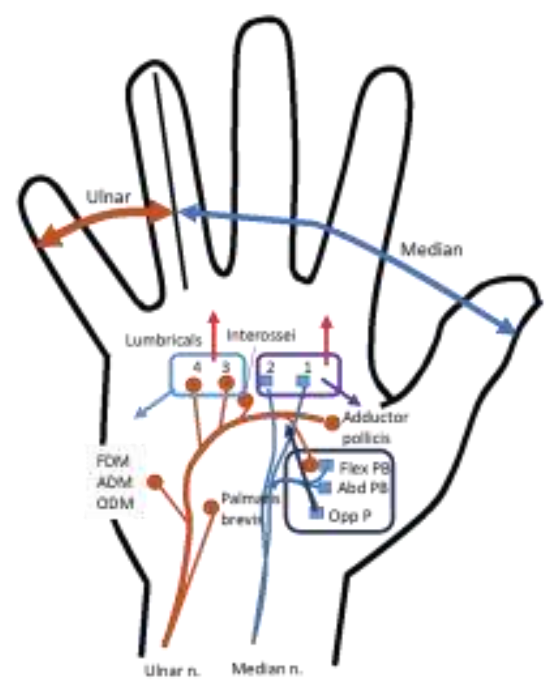

Fig. 7. In overlay to the hand nerve structure, the expected positioning of the $\mathrm{L}$ matrix and of the T matrix. The teal and purple rectangles, $2-3 \mathrm{~cm}$ proximal to the MCP joints, represent the expected position of the $\mathrm{L}$ matrix on the dorsal side of the hand. The counter electrode, not shown, lies on the palm side axially aligned with the transverse palm creases. A distal shift of the L matrix (red arrows) furthers the distance of the active sites from the lumbricals motor points (1-4), thus increasing discomfort (pricking and stinging due to $\mathrm{A} \delta$ fibers activation) and rendering stimulation less effective from a kinematic viewpoint. A proximal shift translates in the central region in unselective stimulation of the ulnar nerve, often reported as tickling or stinging pain with limited or no motor outcome. When the $\mathrm{L}$ electrode array is too large for the hand or intentionally misplaced toward the hypothenar eminence (teal rectangle and arrow) flexion and abduction of the digiti minimi can be triggered by L5, although not clear opposition was achieved. When, conversely, the shift occurs in direction of the thumb (purple rectangle and arrow), co-activation of thumb adduction and index MCP were reported. T matrix: the blue rectangle shows the expected positioning on the thenar eminence. The counter electrode, not shown, is on the volar side the expected abduction and opposition of the thumb. When a shift of the electrode occurred toward the center of the palm (blue arrow), elicitation of the adductor pollicis and of the 1st and 2nd lumbrical could occur, leading to a lateral grip.

could arise in neutral position, electrode arrays were not placed on the hypothenar eminence, thus eliciting of the flexor, abductor and opponens digiti minimi are not directly achievable.

One realization of the scan on the $\mathrm{L}$ matrix (test 1), visible in Fig. 8, shows supraliminal selective stimulation of the fingers without significant effects on the thumb. Symmetrically, one

TABLE II

MAIN EFFECTS OF LIMINAL AND SUPRALIMINAL STIMULATION WITH LUMBRICAL AND THENAR MATRICES

\begin{tabular}{|c|c|c|c|c|c|c|}
\hline \multicolumn{2}{|c|}{ Expected motion } & \multicolumn{5}{|c|}{ Matrix L } \\
\hline Finger & Segment & L1 & L2 & L3 & L4 & L5 \\
\hline Thumb & $\mathrm{CMC}$ & $\operatorname{Ad}_{\mathrm{s}}$ & & & & \\
\hline $1^{\text {st }}$ & MCP & $F_{d}$ & $\mathrm{~F}_{\mathrm{s}}$ & & & \\
\hline $2^{\text {nd }}$ & MCP & $\mathrm{F}_{\mathrm{s}}$ & $F_{d}$ & $\mathrm{~F}_{\mathrm{s}}$ & & \\
\hline $3^{\text {rd }}$ & MCP & & $\mathrm{F}_{\mathrm{s}}$ & $F_{d}$ & $F_{d}$ & $F_{s}$ \\
\hline \multirow[t]{3}{*}{$4^{\text {th }}$} & \multirow[t]{3}{*}{$\mathrm{MCP}$} & & & $\mathrm{F}_{\mathrm{s}}$ & $\mathrm{F}_{\mathrm{s}}$ & $\mathrm{F}_{\mathrm{d}}$ \\
\hline & & & & Matrix & & \\
\hline & & $\mathrm{T} 1$ & $\mathrm{~T} 2$ & T3 & $\mathrm{T} 4$ & T5 \\
\hline Thumb & $\mathrm{CMC}$ & $\mathrm{Op}_{\mathrm{d}}$ & $\mathrm{Op}_{\mathrm{d}}$ & $\mathrm{Op}_{\mathrm{d}}$ & $\mathrm{Op}_{\mathrm{d}}$ & $\mathrm{Op}_{\mathrm{d}}$ \\
\hline Thumb & MCP & Fs & & Fs & & \\
\hline $1^{\mathrm{st}}$ & MCP & Fs & & & & \\
\hline $4^{\text {th }}$ & & & & $\mathrm{Op}_{\mathrm{s}}$ & $\mathrm{Op}_{\mathrm{s}}$ & $\mathrm{Op}_{\mathrm{s}}$ \\
\hline
\end{tabular}

Legend: Flexion (F), Extension (E), Adduction (Ad), Abduction (Ab) Opposition (Op). The subscript indicates if the elicited motor task is dominant (d) or a secondary effect (s). Data extracted from $\mathrm{N}=10$ healthy subjects.

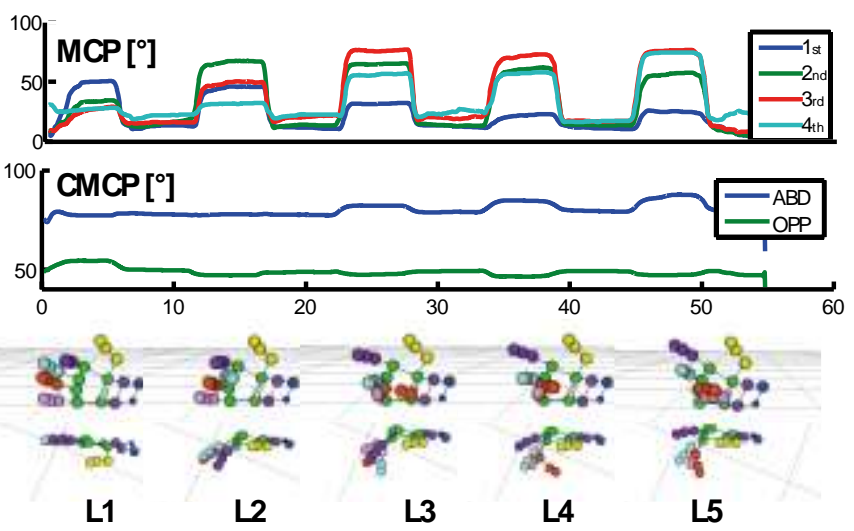

Fig. 8. Stimulation sequence on the L matrix, from index to little finger. Top panel: MCP joint flexion for fingers 1 to 4 . Bottom panel: palmar and top view of the reconstructed hand configuration for each stimulation phase. In the shown fragment, the index ( $1^{\text {st }}$ finger) MCP flexion is elicited by pins L1 (beginning of the sequence) and flexes more than the neighboring fingers, whereas its kinematic response decreases as the active pin shifts toward the ring finger. Likewise, medium finger ( $2^{\text {nd }}$ finger) MCP flexion is dominant when pin $\mathrm{L} 2$ is active. With pin $\mathrm{L} 3$ the ring finger ( ${ }^{\text {rd }}$ finger) activation is dominant and contributes to passively drag neighboring fingers. In the test subject a mechanically coupled activation of MCP and PIP joint of the ring finger can be notice. As the stimulation is applied to pins L4 and L5, index and medium fingers kinematic contribution is reduced, whereas the contribution of the ring finger is maintained constant and the last finger ( $4^{\text {th }}$ finger) is also activated. If properly aligned, no co-activation of the thumb is expected.

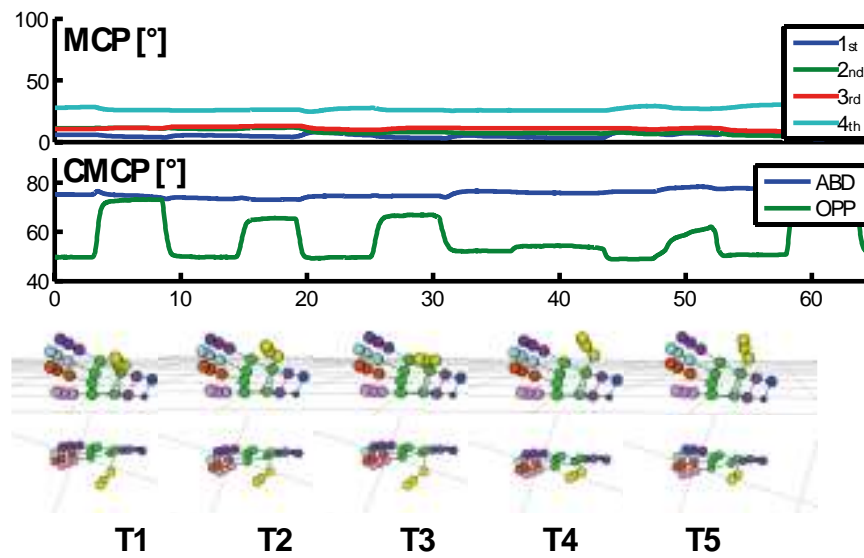

Fig. 9. Stimulation sequence on the T matrix, from thumb to center of the hand palm. Top panel: MCP joint flexion for fingers 1 to 4 . Bottom panel: palmar and top view of the reconstructed hand configuration for each stimulation phase. In the shown fragment, thumb opposition is consistently obtained through the stimulation of pins from T1 to T3, although T2 is the most comfortable. No induced motion is visible on fingers 1 to 4 .

realization of the scan on the $\mathrm{T}$ matrix (test 2), shows supraliminal selective stimulation of the Thumb without significant crosstalk effects on the fingers Fig. 9. Table 2 summarizes the experimental results obtained in case of singlepin optimal stimulation (dominant motion without crossstimulation) and sub-optimal stimulation (misalignment or overstimulation causing the dominant effect and secondary effects). Increased stimulation intensity results in uncontrolled secondary effects or nociceptive sensations. The combined effect of single pin $\mathrm{L}$ and $\mathrm{T}$ stimulation (test 3), visible in Fig. 10 , exemplifies enhanced crosstalk effects when supraliminal stimulation is applied both on thenars and on the lumbricals in proximity of the index (L1). When the distance between the optimal thenar location and of the active $\mathrm{L}$ pin furthers from the index, crosstalk effects reduce on the index, and can arise 

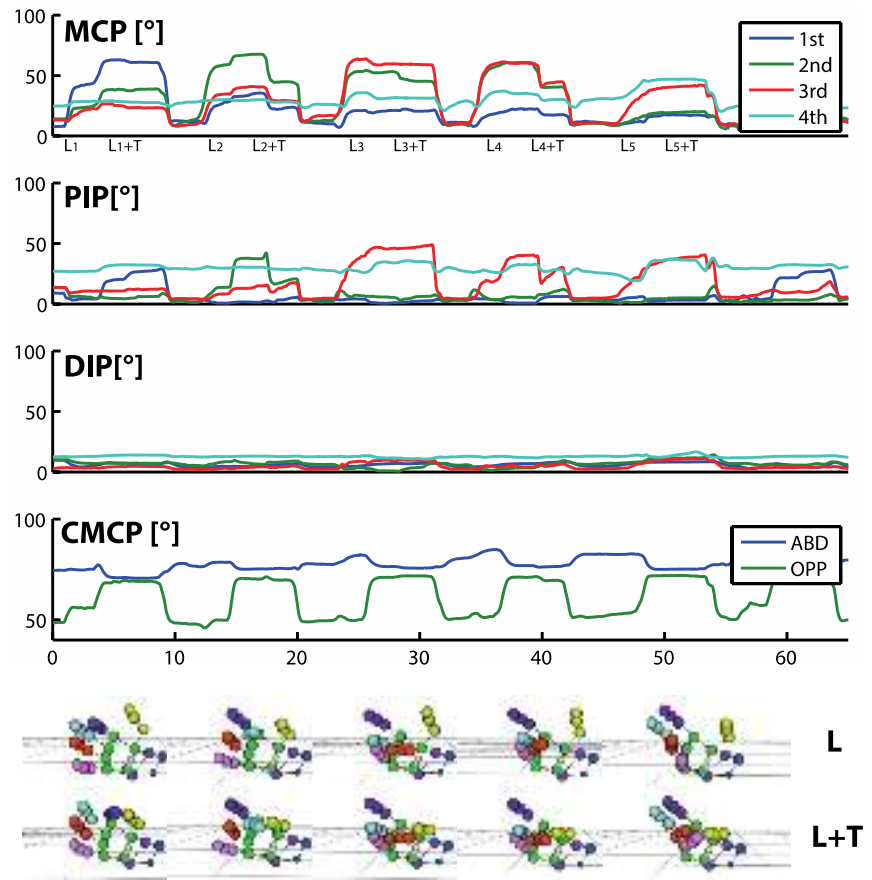
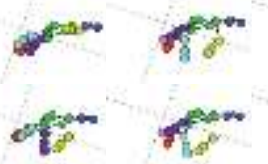

L1

$\mathbf{L 2}$

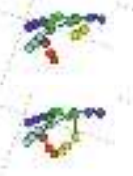

L3

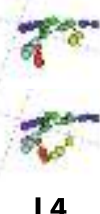

L4

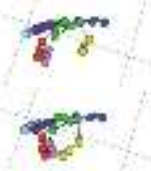

L5
Fig. 10. Stimulation sequence on the L matrix, with delayed onset of stimulation on $\mathrm{T}$ matrix from thumb to center of the hand palm. Top panel: MCP joint flexion for fingers 1 to 4 . Bottom panel: palmar and top view of the reconstructed hand configuration for each stimulation phase. In the shown fragment, thumb opposition is consistently obtained through the stimulation of pins from T1 to T3, although T2 is the most comfortable. No induced motion is visible on fingers 1 to 4 . In this sequence, the combined activation of $\mathrm{L}$ and $\mathrm{T}$ induces thumb-first finger pinching (L1-T), thumb-second finger pinching (L2-T), thumb-third finger pinching (L3-T, or L4-T), and thumbfourth finger pinching (L5-T).

progressively on the other fingers. As described in Fig. 11, synchronous multi-electrode activation (test 4 ) could be used to obtain partial and complete extension grip, as an independent grasp type, or as an intermediate hand posture usable to grant a smooth transition to power grasp, and to reduce the risk of unwanted claw grasp.

Table 3 shows a summary of the grasp types elicited with the standard mounting of the electrode arrays for intrinsic muscles, details the used configurations, and shows the extended set of achieved grasps.

\section{Closed Loop control}

The stimulation setting procedure could result in different selections of pins and different values of NMES parameters; despite these differences, however, the wearable technology achieved a functionally efficient and effective stimulation capable of properly controlling the grasping action. Grasping real objects is not purely a kinematic challenge. It also depends on the chosen affordance for the specific object; for this reason, proper force feedback is needed. In our experiments, the optimal location and current intensity remained constant once
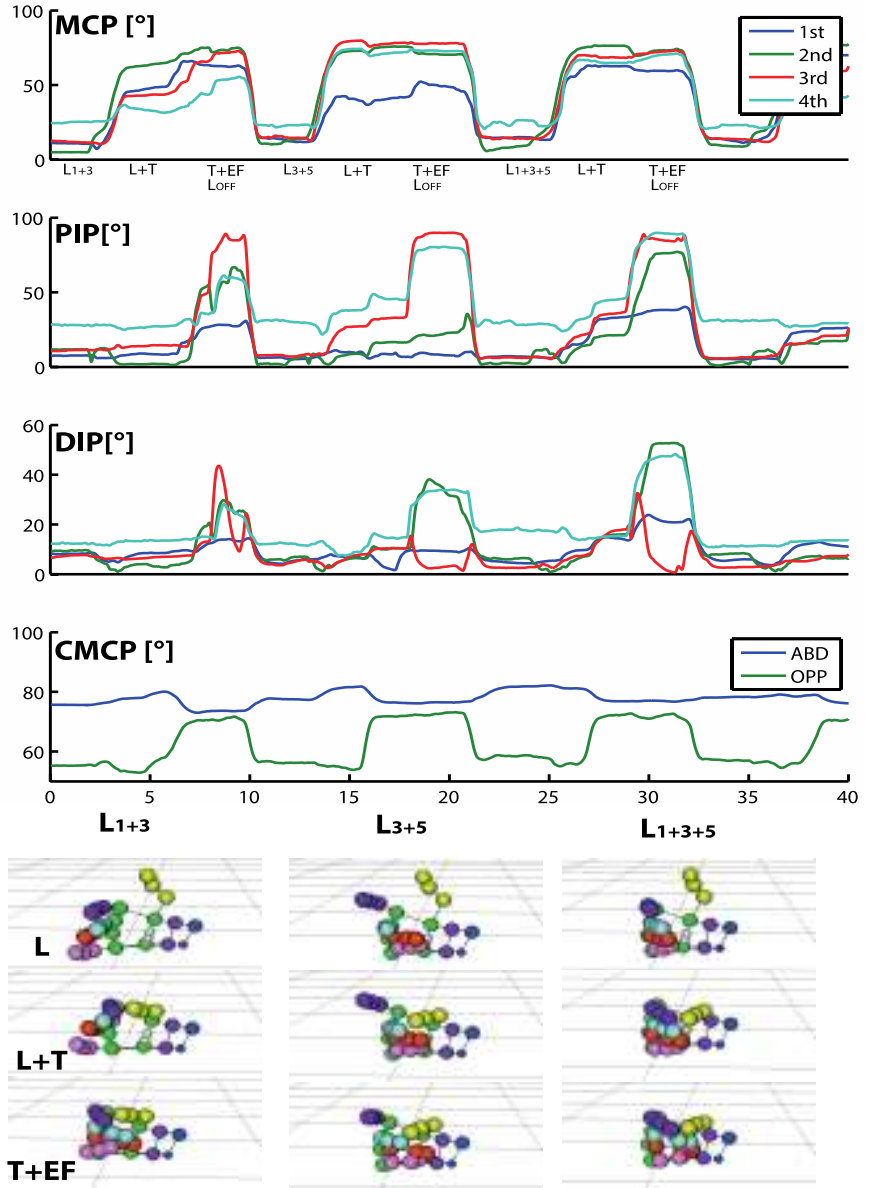

Fig. 11. Stimulation sequence on the L matrix, with three phases coupled activation of pins L1-L3, L3-L5, and L1-L3-L5. In each phase the L array is elicited first, T optimal has a delayed onset of 2.5 seconds. Onset of extrinsic flexors stimulation, delayed of 5 seconds from stimulation start, is synchronized with the deactivation of Lumbrical stimulation. In the first phase, with the activation of the pins L1-L3, lumbricals innervated through the median nerve are synchronously activated, and the thumb opposed to obtain extension grip with fingers one and two. With the activation of the extrinsic flexor, the extension grip is modified into a power grasp with the second and third finger. In the second phase, with the activation of the pins L3-L5, lumbricals innervated through the ulnar nerve are synchronously activated, and the thumb opposed to obtain extension grip with fingers three and four. With the activation of the extrinsic flexor, the extension grip is modified into a power grasp with the third and fourth finger. In the third phase, with the activation of the pins L1-L3-L5, lumbricals innervated through the median and ulnar nerves are synchronously activated, and the thumb opposed to obtain a complete extension grip. With the activation of the extrinsic flexor, the extension grip is modified into a power grasp with the third and fourth finger. The selection of the pins for this realization was found to be dependent on the longitudinal displacement of the L matrix, and on the hand size of the testing subject. For smaller hands, or more proximal positioning of the array, the coupled activation could be obtained with pins L2, L4, L2-L4.

they were set, whereas stimulation was adapted by modulating the pulse width. Four subjects were tested in a repetitive grasp task, hand held in neutral prono-supination position with a cylindrical object $6 \mathrm{~cm}$ wide. Stimulation was set only on extrinsic flexors in a pre-determined comfortable location aimed at providing selective flexion of the ring flexion or, if not possible, of ring and medium finger. During the identification, pulsewidth was set to $300 \mu \mathrm{s}$, and the intensity of current empirically chosen to exert the maximum comfortable grasp. Stimulations triggering wrist flexion alongside with the fingers flexion were excluded, thus restricting optimal and close to 

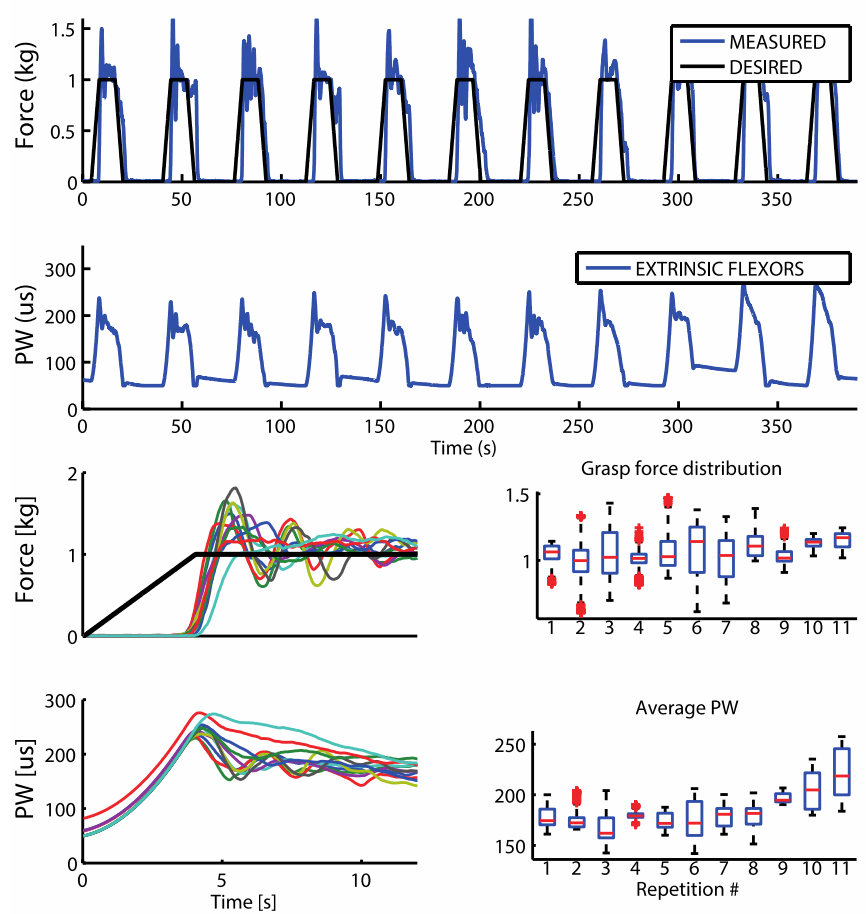

Fig. 12. From top to bottom. First panel: desired (black) and measured (blue) grasp force profiles. Second panel: applied pulsewidth modulation profile; the range is limited between 50 and $300 \mu$ s. The stimulation is switched off when the pulsewidth reaches the lower bound. Third panel, left: desired force and synchronized motor response. Third panel, right: distribution of the exerted force during each grasp realization, data from $6 \mathrm{~s}$ to $12 \mathrm{~s}$. Fourth panel left: synchronized pulsewidth output. Fourth panel, right: distribution of the pulsewidth during each grasp realization. In this grasp sequence, the desired force is reached with low pulsewidth. Fatigue onset is visible staring from the 9th shown repetition.

optimal locations to pins 3 and 4 on MD, and 7, 8, 11, 12, 16, and 17 on MP. These location also reflected the ones used for the final test of grasp sequencing. The force setpoint during the repeated grasp tasks was chosen as lower or equal to the $60 \%$ of the maximum grasp intensity elicited during the identification. This controller was designed not to cope with finer grasp tasks, shown in the previous section, but rather tasks in which sufficient force is required to hold and lift common non-fragile objects usable in daily activities. The controller was tested with three subjects naïve to electrical stimulation, and with three subjects already comfortable with it. Fig. 11 shows the results on a subject able to exert medium-large forces,
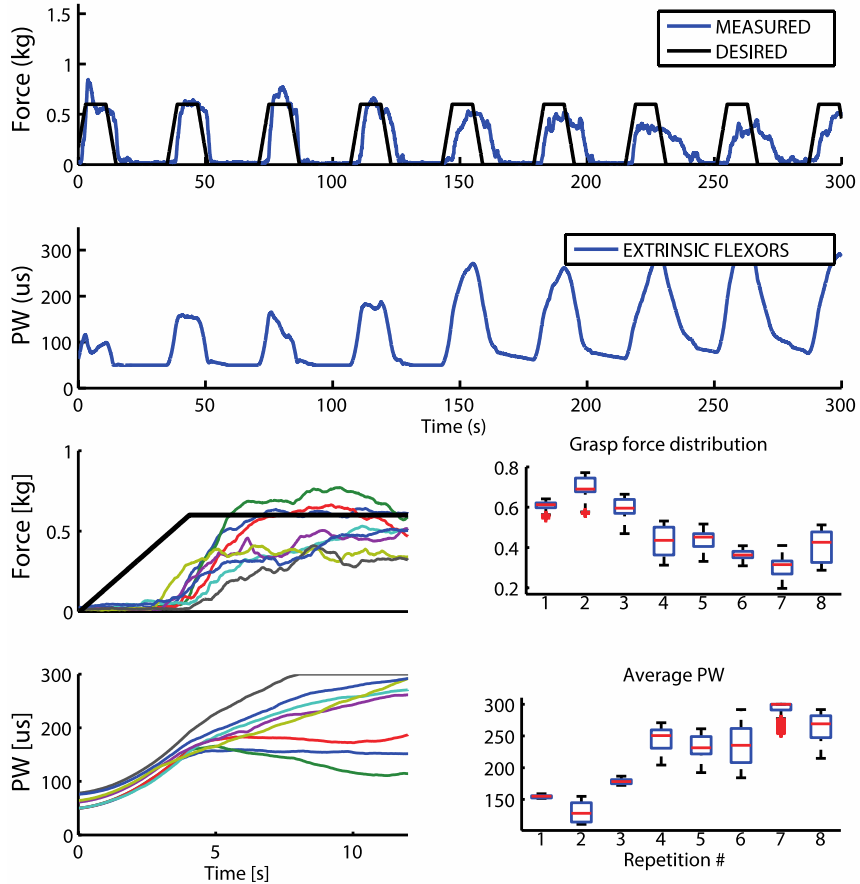

Fig. 13. For panels description please refer to Fig. 12. The subject is naive to stimulation and prone to fatigue. In the shown fragment, the desired force is not reachable at low stimulation intensities. A quick increment of the average pulsewidth is visible across the repetitions.

comfortable with NMES, and less prone to muscle fatigue. Fig. 12 exemplifies a subject with a muscle structure able to exert low to medium grasp forces, naïve to electrical stimulation, and prone to quick muscle fatigue. The desired force was set to $1 \mathrm{~kg}$ for the larger subject, and to $0.6 \mathrm{~kg}$ for the thinner subject. As visible in the Grasp force distribution subpanels, the desired grasp force was consistently achieved with the first subjects, with the pulsewidth stable and slowly increasing to adapt to the fatigue onset. The naïve subject, on the other side, was more subject to a quick fatigue onset and less capable to relax and mimic a flaccid hand, thus leading to a less consistent grasp output.

\section{DISCUSSIONS AND CONCLUSIONS}

NMES can be used to restore grasping function in impaired subjects. The goal of our study was to develop a novel wearable NMES system with multiple arrays that could serve as a

TABLE III

GRASP TYPES, MAIN KINEMATIC REQUIREMENTS, AND USABLE CONFIGURATIONS

\begin{tabular}{|c|c|c|c|c|c|c|c|}
\hline \multirow{2}{*}{$\begin{array}{l}\text { Grasp } \\
\text { Type }\end{array}$} & \multirow{2}{*}{$\begin{array}{c}\text { Independence } \\
{[\%]}\end{array}$} & \multirow{2}{*}{$\begin{array}{l}\text { Thumb } \\
\text { opposition } \\
\text { required }\end{array}$} & \multicolumn{3}{|c|}{ Hand Arches } & \multicolumn{2}{|c|}{ Grasp obtained with } \\
\hline & & & Distal & Oblique & Longitudinal & Extrinsic only & Extrinsic + Intrinsic \\
\hline \multirow[t]{2}{*}{ Power } & 25 & $\begin{array}{c}\text { No } \\
\text { (small } \\
\text { objects) }\end{array}$ & & & Yes & $\begin{array}{c}\text { Yes } \\
\text { (MP/MD) }\end{array}$ & $\begin{array}{c}\text { Yes } \\
(\mathrm{MP} / \mathrm{MD} / \mathrm{T})\end{array}$ \\
\hline & & $\begin{array}{l}\text { Yes } \\
\text { ( large } \\
\text { objects) }\end{array}$ & & & & No & $\begin{array}{c}\text { Yes } \\
(\mathrm{L}+\mathrm{T}+\mathrm{MP} / \mathrm{MD})\end{array}$ \\
\hline Tip & 22 & Yes & & & Yes & No & $\begin{array}{c}\text { Yes } \\
(\mathrm{L}+\mathrm{T}+\text { mechanical } \\
\text { constraining })\end{array}$ \\
\hline Lateral & 21 & Yes & & Yes & Yes & No & $\begin{array}{l}\text { Yes (shifted Thenar } \\
\text { or shifted Lumbrical) }\end{array}$ \\
\hline Spherical & 10 & Yes & Yes & Yes & Yes & No & No \\
\hline Tripod & 8 & Yes & & & Yes & No & Yes $\left(\mathrm{L}_{12}+\mathrm{T}_{\text {optimal }}\right)$ \\
\hline Extension & 8 & & & & Yes & No & Yes $\left(L_{135}\right.$ or $\left.L_{24}\right)$ \\
\hline Other & 6 & & & & & No & \\
\hline
\end{tabular}


modular tool suitable for use as a platform for grasp rehabilitation, potentially improving the clinical applicability of NMES. The system targets both extrinsic and intrinsic hand grasp muscles, which is potentially very promising for improving its clinical efficacy and flexibility. The hardware structure can also be easily customized.

The validation experiments described in the manuscript were designed to highlight the flexibility of our device, showing how different results can be achieved using different electrodes on each pad for each subject. To better characterize these differences, we used a camera-based system to obtain the most accurate kinematic measurements. However, a simpler (but in any case effective) characterization could be developed by therapists in other ways that are more compatible with a clinical setting. For example, one possible approach able to provide clinically intuitive calibration relies on the creation of stimulation maps, which allows to configure location, intensity and active electrode shape by means of a simple graphical user interface hosted on a touch-compliant screen [27]-[29].

The garment represents an improvement beyond the two previous wearable NMES systems recently developed by Lawrence et al. [7] and by Malesevic et al. [5]. The embroidered EAs developed by Lawrence in collaboration with Bischoff Textile (St. Gallen, $\mathrm{CH}$ ) featured active elements embedded in the fabric. Conductive yarn embroidery on fabric offers adaptable stretchability, but conductive yarns are prone to breaking under stress; multiple stitching is required to maintain conductivity at the cost of a larger and thicker footprint. Malesevic's thin array exhibited very good electrical properties, better electrical isolation, and higher routing density, but it was characterized as a general-purpose design.

We have developed a tool that is adaptable to different forearm sizes, both in length and circumference, and potentially applicable to clinical routines. The garment for NMES presented in this study embeds EAs and can exploit spatial and temporal stimulation patterns (i.e., a set of stimulation electrodes coupled with a set of stimulation intensities assigned to individual electrodes) for grasp restoration, supported by a real-time adaptable stimulator device. In contrast to current commercial and research NMES systems, our garment makes use of multiple sets of EAs that are shaped to conform to the hand and forearm. Hand intrinsic movements can be elicited by stimulating the thenar muscles, lumbricals and palmar interossei. Extrinsic muscles of the hand, when elicited by stimulation, allow flexion and extension of fingers, thumb adduction, wrist flexion/extension and ulnar/radial deviation. Because the garment is divided into six different matrices, each of them adaptable in size if needed, subsets of functionalities can be added simply by selecting a subset of matrices through the main PCB. Patients needing to train only thumb adduction and finger extension can benefit by using only the corresponding matrices LP and LD; patients requiring selective training or support for the intrinsic hand muscles can use the $\mathrm{T}$ and $\mathrm{L}$ matrices, and patients requiring support in more complex conditions can benefit from the full configuration.

The controls allow effective kinematic-based hand preshaping and grasping with force feedback. The current design implementation of the HandNMES system allows control of simple hand grasps. The closed-loop experiments are presented here as a case study, but the glove can be easily replaced with sensorized objects or hand orthoses embedded artificial sensors. Specifically, for individuals with contractures or spasticity, splints or hand orthoses can be used to constrain the hand in its intended use. Constraining implies that some rigidity in the device is necessary; thus, residual sensory feedback is reduced, as are available motions. The variability of splinting needs is mitigated clinically by the partial customizability of each commercially available device. Sensorized clasps can be adapted to operate with the chosen splint, or splints can be designed alongside the clasps, but the design requirements rely on the clinically chosen functional constraints and on an acceptable trade-off between reliable force detection, overall encumbrance, and acceptable sensory masking.

\section{A. Limits of the device}

The current generation of our device also has some drawbacks solvable by redesign; for example, the PCB-arrays connector gates were designed for compactness, and a misalignment between connector and contact translates into poor contact conditions. This fault can appear when consistent shear stress is applied to the garment or when an impact occurs on the rigid PCB. The EAs exhibited good resistance to repetitive bending, but extreme curvature can cause permanent damage to the disposable EAs. This scenario occurs, for example, when extremely thin subjects don the garment and the array is abruptly pulled and bent at $90^{\circ}$. From a usability perspective, gel patches are prone to quick deterioration because of donning/doffing stress and because of dehydration and need to be replaced, on average, after 10 hours of stimulation. Removing gel patches from the EA induces moderate mechanical stress on the EAs and thus progressively damages them during each removal operation. EAs can empirically be used through 8-40 gel patch replacements.

\section{B. Next steps}

In the coming months, further developments will be incorporated to improve the performance of this technology. For example, a more intuitive user interface will be developed to allow use of the garment as-is in clinical testing and to empower clinicians with a variety of cyclic stimulations with contralaterally controlled or BCI controlled paradigms. Finally, as the garment focuses only on hand functions, it could be used together with upper limb exoskeletons to achieve functional (reaching and grasping) tasks[30]. A controller, implemented by means of a finite state machine, can adapt the stimulation patterns in real time. Depending on the complexity of the desired grasp task and the responsiveness of the user to stimulation, different intermediate states can be implemented. Specific tasks can often be split into sub phases; e.g., relax, hand open, and grasp. In some conditions, such as hyper responsiveness to extrinsic flexor stimulation, complete PIP joint flexion is achieved without prior MCP joint accommodation, making grasping unfeasible. By introducing an additional hand pre-shape phase, in which only intrinsic hand muscles are elicited, claw grasp issues can be mitigated. Finally, the HandNMES system will be tested in clinical trials employing patients affected by neurological disorders. 


\section{ACKNOWLEDGMENT}

The work was partly supported by the EU MUNDUS grant ICT-2009.7.2 (Accessible and Assistive ICT GA 248326), the Swiss National Centre of Competence in Research (NCCR) Robotics, the Foundations "Fondazione CARIPLO" of Milan, Italy, and "CariGest" of Geneva, Switzerland (Project "INCOGNITO"), the EU RETRAINER. Project ID: 644721. (H2020-EU.2.1.1.5) - and the Bertarelli Foundation. We thank Robert Nguyen for the help provided and also all the subjects who agreed to participate in the study.

\section{REFERENCES}

[1] "Bioness Inc. - H200 for Hand Paralysis." [Online]. Available: http://www.bioness.com/Products/H200_for_Hand_Paralysis.php. [Accessed: 11-Sep-2016].

[2] A. Prochazka, M. Gauthier, M. Wieler, and Z. Kenwell, "The bionic glove: An electrical stimulator garment that provides controlled grasp and hand opening in quadriplegia," Arch. Phys. Med. Rehabil., vol. 78, no. 6, pp. 608-614.

[3] R. R. Kaliki, R. Davoodi, and G. E. Loeb, "Evaluation of a Noninvasive Command Scheme for Upper-Limb Prostheses in a Virtual Reality Reach and Grasp Task," IEEE Trans. Biomed. Eng., vol. 60, no. 3, pp. 792-802, Mar. 2013.

[4] Keller, B. Hackl, M. Lawrence, and A. Kuhn, "Identification and control of hand grasp using multi-channel transcutaneous electrical stimulation," 2006 .

[5] N. M. Malesevic et al., "A multi-pad electrode based functional electrical stimulation system for restoration of grasp," $J$. NeuroEngineering Rehabil., vol. 9, no. 1, p. 66, Sep. 2012.

[6] U. Hoffmann, M. Deinhofer, and T. Keller, "Automatic determination of parameters for multipad functional electrical stimulation: application to hand opening and closing," Conf. Proc. Annu. Int. Conf. IEEE Eng. Med. Biol. Soc. IEEE Eng. Med. Biol. Soc. Annu. Conf., vol. 2012, pp. 1859-1863, 2012.

[7] D. B. Popović and M. B. Popović, "Automatic determination of the optimal shape of a surface electrode: selective stimulation," $J$. Neurosci. Methods, vol. 178, no. 1, pp. 174-181, Mar. 2009.

[8] L. Popović-Maneski et al., "Multi-pad electrode for effective grasping: design," IEEE Trans. Neural Syst. Rehabil. Eng. Publ. IEEE Eng. Med. Biol. Soc., vol. 21, no. 4, pp. 648-654, Jul. 2013.

[9] M. Lawrence, Transcutaneous Electrode Technology for Neuroprostheses. lulu.com, 2015.

[10] A. Popović-Bijelić, G. Bijelić, N. Jorgovanović, D. Bojanić, M. B. Popović, and D. B. Popović, "Multi-field surface electrode for selective electrical stimulation," Artif. Organs, vol. 29, no. 6, pp. 448-452, Jun. 2005

[11] K. J. Hunt, J. Fang, J. Saengsuwan, M. Grob, and M. Laubacher, "On the Efficiency of FES Cycling: A Framework and Systematic Review," Technol Health Care, vol. 20, no. 5, pp. 395-422, Sep. 2012.

[12] N. M. Malesević, L. Z. Popović, L. Schwirtlich, and D. B. Popović, "Distributed low-frequency functional electrical stimulation delays muscle fatigue compared to conventional stimulation," Muscle Nerve, vol. 42, no. 4, pp. 556-562, Oct. 2010.

[13] L. Z. P. Maneski, N. M. Malešević, A. M. Savić, T. Keller, and D. B. Popović, "Surface-distributed low-frequency asynchronous stimulation delays fatigue of stimulated muscles," Muscle Nerve, vol. 48, no. 6, pp. 930-937, Dec. 2013

[14] R. Nguyen, K. Masani, S. Micera, M. Morari, and M. R. Popovic, "Spatially Distributed Sequential Stimulation Reduces Fatigue in Paralyzed Triceps Surae Muscles: A Case Study," Artif. Organs, vol. 35, no. 12, pp. 1174-1180, Dec. 2011

[15] D. G. Sayenko, R. Nguyen, M. R. Popovic, and K. Masani, "Reducing muscle fatigue during transcutaneous neuromuscular electrical stimulation by spatially and sequentially distributing electrical stimulation sources," Eur. J. Appl. Physiol., vol. 114, no. 4, pp. $793-$ 804, Apr. 2014.

[16] D. B. Popović, "Advances in functional electrical stimulation (FES)," J. Electromyogr. Kinesiol., vol. 24, no. 6, pp. 795-802, Dec. 2014.

[17] A. Kuhn, T. Keller, M. Lawrence, and M. Morari, "The influence of electrode size on selectivity and comfort in transcutaneous electrical stimulation of the forearm," IEEE Trans. Neural Syst. Rehabil. Eng. Publ. IEEE Eng. Med. Biol. Soc., vol. 18, no. 3, pp. 255-262, Jun. 2010.

[18] A. Pedrocchi et al., "MUNDUS project: MUltimodal neuroprosthesis for daily upper limb support," J. Neuroengineering Rehabil., vol. 10, p. 66, 2013.

[19] "hart toolbox." [Online]. Available: http://hart.sourceforge.net/. [Accessed: 06-Mar-2017].

[20] A. Barre and S. Armand, "Biomechanical ToolKit: Open-source framework to visualize and process biomechanical data," Comput. Methods Programs Biomed., vol. 114, no. 1, pp. 80-87, Apr. 2014.

[21] M. Santello, M. Flanders, and J. F. Soechting, "Postural Hand Synergies for Tool Use," J. Neurosci., vol. 18, no. 23, pp. 10105-10115, Dec. 1998.

[22] A. P. Sangole and M. F. Levin, "Arches of the hand in reach to grasp," J. Biomech., vol. 41, no. 4, pp. 829-837, 2008.

[23] C. Sollerman and A. Ejeskär, "Sollerman hand function test. A standardised method and its use in tetraplegic patients," Scand. J. Plast. Reconstr. Surg. Hand Surg., vol. 29, no. 2, pp. 167-176, Jun. 1995.

[24] C. M. Light, P. H. Chappell, and P. J. Kyberd, "Establishing a standardized clinical assessment tool of pathologic and prosthetic hand function: Normative data, reliability, and validity," Arch. Phys. Med. Rehabil., vol. 83, no. 6, pp. 776-783, Jun. 2002.

[25] U. Arnet, D. A. Muzykewicz, J. Fridén, and R. L. Lieber, "Intrinsic Hand Muscle Function, Part 1: Creating a Functional Grasp," J. Hand Surg., vol. 38, no. 11, pp. 2093-2099, Nov. 2013.

[26] D. A. Muzykewicz, U. Arnet, R. L. Lieber, and J. Fridén, "Intrinsic Hand Muscle Function, Part 2: Kinematic Comparison of 2 Reconstructive Procedures," J. Hand Surg., vol. 38, no. 11, p. 21002105.e1, Nov. 2013.

[27] A. Crema et al., "The RETRAINER hand orthosis: an event-driven modular lightweight device that combines controlled hand-wrist motion and multi-electrode neuromuscular stimulation," in Proceeding of the 12th Vienna International Workshop on FES IFESS, Vienna, 2016.

[28] J. Zajc et al., "Unified Control Interface for multiple Devices used for Upper Limb Rehabilitation after Stroke," in 12th Vienna International Workshop on FES, Vienna.

[29] A. Crema et al., "Helping Hand grasp rehabilitation: preliminary assessment on chronic stroke patients," in 2017 8th International IEEE/EMBS Conference on Neural Engineering (NER), Shanghai.

[30] A. Crema, M. Mancuso, A. Frisoli, F. Salsedo, F. Raschella, and S. Micera, "A hybrid NMES-exoskeleton for real objects interaction," in 20157 th International IEEE/EMBS Conference on Neural Engineering (NER), 2015, pp. 663-666.

Andrea Crema received his Ph.D. degree in Electrical Engineering (2017) from the Ecole Polytechnique Fédérale de Lausanne. He is currently postdoc at the Translational Neural Engineering Laboratory, EPFL, Campus Biotech, Geneva, where he focuses on design of hybrid hardware software systems for upper extremity rehabilitation, skin-contact technologies for sensing and stimulation, and environmental sensing and interaction. He has been working in the following international projects: Retrainer EU-project, Mundus EUProject, NCCR SmartArmeo and ReHand, Incognito, EchordLinarm++.

Nebojša Malešević received the Ph.D. degree in Biomedical engineering in 2014 from the Faculty of Electrical Engineering, University of Belgrade, Serbia. He is currently a Postdoc at the Department of Biomedical Engineering, Lund University and a Researcher at the Faculty of Electrical Engineering, University of Belgrade. His research interests are the control of movement strategies, functional electrical stimulation intended for lower and upper extremity rehabilitation and prosthetic limbs with advanced interfacing between neural system and artificial system. 
Ivan Furfaro received the B.S. and M.S. degrees (cum laude) from the Biomedical Engineering faculty at Politecnico di Torino, Italy, in 2013 and 2015 respectively. His Master Thesis dealt with the design and validation of an EMG Low-Power recording system. In 2016 he worked as Scientific Assistant in the Translational Neural Engineering Laboratory, EPFL, Lausanne, he was involved in a European project aimed to design rehabilitation system for stroke survivors. Currently, he is working as Electrical Engineer in the Laboratory for Soft Bioelectronic Interfaces, Campus Biotech, Genève where he designs hardware/software systems to evaluate the integration of soft interfaces

Flavio Raschellà received the B.Sc. degree in mechanical engineering from the La Sapienza University, Rome, Italy, in 2012, and the M.Sc. degree in biomedical engineering from the Campus Bio-Medico University, Rome, Italy, in 2014. He is currently working toward the Ph.D. degree in the electrical engineering at the Ecole polytechnique fédérale de Lausanne, Lausanne, Switzerland, where he is focusing on design, development, and validation of an algorithmic approach for estimation of spinal cord stimulation protocols for restoration of locomotion after spinal cord injury or Parkinson's disease.

Alessandra Pedrocchi received the M.S. degree in electrical engineering (1997) and the Ph.D. in bioengineering (2001) from the Politecnico di Milano. She is an Associate Professor at the Department of Electronics, Informatics and Bioengineering of the Politecnico di Milano, teaching Neuroengineering to MA students and Biomedical Instrumentation to BS students. She works in the NeuroEngineering and Medical Robotics laboratory, investigating neurorobotics, computational neuroscience, and advanced technologies for neurorehabilitation. She has been working in the following international projects: Retrainer EUproject, REALNET EU-project and Multi-center trial of Augmented Sensory Feedback in Children with Dyskinetic CP NIH-project.

Silvestro Micera is currently Professor of Biomedical Engineering at the Scuola Superiore Sant'Anna (SSSA, Pisa, Italy), and Associate Professor of Biomedical Engineering at the Ecole Polytechnique Federale de Lausanne (Lausanne, Switzerland) where he is holding the Bertarelli Foundation Chair in Translational NeuroEngineering. He received the University degree (Laurea) in Electrical Engineering from the University of Pisa, in 1996, and the Ph.D. degree in Biomedical Engineering from the Scuola Superiore Sant'Anna, in 2000. From 2000 to 2009, he has been an Assistant Professor of BioRobotics at the Scuola Superiore Sant'Anna where he is now Professor and the Head of the Translational Neural Engineering Area. In 2007 he was a Visiting Scientist at the Massachusetts Institute of Technology, Cambridge, USA with a Fulbright Scholarship. From 2008 to 2011 he was the Head of the Neuroprosthesis Control group and an Adjunct Assistant Professor at the Institute for Automation, Swiss Federal Institute of Technology, Zurich, CH. In 2009 he was the recipient of the "Early Career Achievement Award" of the IEEE Engineering in Medicine and Biology Society. From 2011 he is Associate Professor and Head of the Translational Neural Engineering Laboratory at the EPFL. Dr. Micera's research interests include the development of neuroprostheses based on the use of implantable neural interfaces with the central and peripheral nervous systems to restore sensory and motor function in disable persons. In particular, he is currently involved in translational experiments for hand prosthesis control in amputees, and the restoration of vestibular function, grasping and locomotion in different neurological disorders. He is author of more than 100 ISI scientific peer-reviewed papers and several international patents. He is currently Associate Editor of IEEE Transactions on Neural Systems and Rehabilitation Engineering. He is also member of the Editorial Boards of the Journal of Neuroengineering and Rehabilitation, of Journal of Neural Engineering, and of the IEEE Journal of Translational Engineering in Health and Medicine.received the B.Sc. degree in mechanical engineering from the La Sapienza University, Rome, Italy, in 2012, and the M.Sc. degree in biomedical engineering from the Campus Bio-Medico University, Rome, Italy, in 2014. He is currently working toward the Ph.D. degree in the electrical engineering at the Ecole polytechnique fédérale de Lausanne, Lausanne, Switzerland, where he is focusing on design, development, and validation of an algorithmic approach for estimation of spinal cord stimulation protocols for restoration of locomotion after spinal cord injury or Parkinson's disease. 